\title{
The impact of anthracyclines in intermediate and high-risk HER2-negative early breast cancer-a pooled analysis of the randomised clinical trials PlanB and SUCCESS $C$
}

\author{
Amelie de Gregorio $\mathbb{D D}^{1 凶}$, Wolfgang Janni (D) ${ }^{1}$, Thomas W. P. Friedl (DD ${ }^{1}$, Ulrike Nitz ${ }^{2,3}$, Brigitte Rack ${ }^{1}$, Andreas Schneeweiss ${ }^{4}$, \\ Ronald Kates ${ }^{3}$, Tanja Fehm ${ }^{5}$, Hans Kreipe ${ }^{6}$, Matthias Christgen ${ }^{6}$, Sherko Kümmel ${ }^{3,7,8}$, Elisabeth Trapp ${ }^{9}$, Rachel Wuerstlein ${ }^{3,10}$, \\ Andreas Hartkopf ${ }^{11}$, Michael Clemens ${ }^{12}$, Toralf Reimer ${ }^{13}$, Lothar Häberle ${ }^{14}$, Peter A. Fasching (D) ${ }^{14}$, Oleg Gluz ${ }^{2,3}$ and Nadia Harbeck $^{3,10}$
}

(c) The Author(s) 2022

BACKGROUND: Anthracycline/cyclophosphamide-taxane-containing chemotherapy (AC-T) is the standard of care in the adjuvant treatment of HER2-negative early breast cancer $(E B C)$, but recent studies suggest omission of anthracyclines for reduced toxicity without compromising efficacy.

METHODS: Based on individual patient data $(n=5924)$ pooled from the randomised Phase III trials PlanB and SUCCESS C, we compared disease-free survival (DFS) and overall survival (OS) between intermediate to high-risk HER2-negative EBC-patients treated with either six cycles of docetaxel/cyclophosphamide (TC6) or an AC-T regime using univariable and adjusted multivariable Cox regression models.

RESULTS: AC-T conferred no significant DFS or OS advantage in univariable (DFS: hazard ratio (HR) for TC vs. AT 1.05, 95\% confidence interval (Cl): 0.89-1.24, $P=0.57$; OS: HR 1.00, 95\% Cl: $0.80-1.26, P=1.00)$ and adjusted multivariable analysis (DFS: HR $1.01,95 \% \mathrm{Cl}: 0.86-1.19, P=0.91$; OS: HR $0.97,95 \% \mathrm{Cl}: 0.77-1.22, P=0.79$ ). Patients receiving TC6 had significantly fewer grade $3-4$ adverse events. Exploratory subgroup analysis showed that AC-T was associated with significantly better DFS and OS in pN2/3 patients, specifically in those with lobular histology.

CONCLUSION: For most patients with HER2-negative EBC, AC-T is not associated with a survival benefit compared to TC6. However, patients with lobular pN2/pN3 tumours seem to benefit from anthracycline-containing chemotherapy.

British Journal of Cancer (2022) 126:1715-1724; https://doi.org/10.1038/s41416-021-01690-6

\section{INTRODUCTION}

Current international guidelines recommend anthracycline/taxane-based chemotherapy as the standard cytotoxic regime for human epidermal growth factor receptor 2 (HER2)-negative early breast cancer (EBC) [1]. Anthracycline-free regimes are currently restricted to patients unwilling or unable to be treated with anthracyclines, e.g., because of cardiac comorbidities or in patients with lower-risk disease.

In 2003, Henderson et al. showed that the addition of sequential paclitaxel to the adjuvant anthracycline-containing chemotherapy (AC) improved outcomes in patients with node-positive primary breast cancer (5-year disease-free survival (DFS) rate for AC plus paclitaxel $70 \%$ vs $65 \%$ for $A C$, 5 -year overall survival (OS) rate $80 \%$ vs $77 \%$ ). However, no benefit was seen with escalation of doxorubicin dose [2]. These findings were supported by the results of the randomised PACS01 trial in 2006, where 1999 patients with node-positive early breast cancer were treated with either six cycles of FEC (fluorouracil, epirubicin, and cyclophosphamide) or three cycles of FEC followed by three cycles of docetaxel. The outcome was better in the taxane-containing treatment group with a 5 -year DFS rate of $78.4 \%$ vs $73.2 \%$ and a 5 -year OS rate of $90.7 \%$ vs $86.7 \%$ [3].

A meta-analysis from the Early Breast Cancer Trialists' Collaborative Group (EBCTCG) compared the long-term outcome of 123

\footnotetext{
${ }^{1}$ Department of Gynecology and Obstetrics, Ulm University Hospital, Ulm, Germany. ${ }^{2}$ Department of Gynecology and Obstetrics, Evangelical Hospital Bethesda, Moenchengladbach, Germany. ${ }^{3}$ Westdeutsche Studiengruppe (WSG), Moenchengladbach, Germany. ${ }^{4}$ National Center for Tumor Diseases, University Hospital and German Cancer Research Center, Heidelberg, Germany. ${ }^{5}$ Department of Gynecology and Obstetrics, Duesseldorf University Hospital, Heinrich-Heine University, Duesseldorf, Germany. ${ }^{6}$ Institute of Pathology, Medical School Hannover, Hannover, Germany. ${ }^{7}$ Breast Unit, Kliniken Essen-Mitte, Essen, Germany. ${ }^{8}$ Clinic for Gynecology with Breast Center, CharitéUniversitaetsmedizin Berlin, Berlin, Germany. ${ }^{9}$ Department of Gynecology and Obstetrics, University of Graz, Graz, Austria. ${ }^{10}$ Breast Center, Department of Gynecology and Obstetrics and CCC Munich, LMU University Hospital, Munich, Germany. ${ }^{11}$ Department of Gynecology and Obstetrics, Tuebingen University Hospital, Tuebingen, Germany.

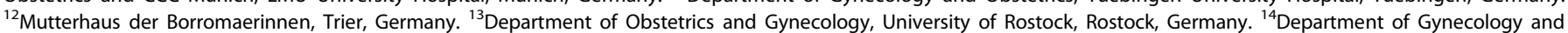
Obstetrics, Erlangen University Hospital, Friedrich-Alexander-University of Erlangen-Nuremberg, Comprehensive Cancer Center EMN, Erlangen, Germany.

email: amelie.degregorio@uni-ulm.de
} 
randomised trials for women with early breast cancer based on different polychemotherapeutic regimes. Based on a cohort of 11.167 patients, the effect of adding four cycles of taxanes to anthracyclines (experimental arm with prolonged treatment duration) was compared to the standard arm with anthracyclinecontaining chemotherapy only. The 8 -year recurrence rate was $30.2 \%$ for the taxane-group vs $34.8 \%$ for the anthracycline-control arm (RR $0.84 ; 96 \% \mathrm{Cl}: 0.78-0.91$ ), the 8-year breast cancer mortality was $21.1 \%$ vs $23.9 \%$ (RR $0.86 ; 95 \% \mathrm{Cl}: 0.79-0.93$ ) and 8 -year overall mortality was $23.5 \%$ vs $26.7 \%$ (RR $0.86 ; 95 \%$ Cl: $0.79-0.93$ ). Based on additional findings of their large meta-analysis, the authors concluded that the 10-year risk of breast cancer mortality can be reduced by on average about one-third with taxane/anthracycline-based chemotherapy regimens or higher-cumulative-dosage anthracycline-based regimens compared to no chemotherapy [4].

Taxane-containing anthracycline-free adjuvant chemotherapy offers an additional evidence-based therapy option for selected early breast cancer patients. Jones et. reported in 2006 the first results of a randomised Phase III trial comparing four cycles of TC (docetaxel cyclophosphamide) or four cycles of standard-dose AC in more than 1000 early breast cancer patients. Both 5-year DFS rate and 5-year OS rate were significantly higher for TC than for AC $(86 \%$ vs 80 and $90 \%$ vs $87 \%$, respectively) [5]. These findings were later confirmed based on even longer follow-up (DFS $81 \%$ TC vs 75\% AC and OS $87 \%$ TC vs $82 \%$ AC) [6], allowing to offer an anthracyclinefree less cytotoxic therapy regimen to lower-risk patients. Nevertheless, the impact of anthracyclines needs to be addressed not only for low-risk but also for high-risk early breast cancer patients for avoiding unnecessary severe long-term toxicities through anthracyclines like acute myeloid leukaemia or cardiotoxicity $[7,8]$.

In 2017, Blum et al. presented a joint efficacy analysis from the three adjuvant $A B C$ trials (US Oncology Research (USOR) 06-090, National Surgical Adjuvant Breast and Bowel Project (NSABP) B-46I/USOR 07132 and NSABP B-49) and concluded that anthracycline/ taxane-based chemotherapy improved invasive DFS in patients with high-risk HER2-negative breast cancer compared to TC6 because the obtained hazard ratio exceeded the prespecified inferiority boundary [9]. Based on current knowledge, taxanes should be considered as therapy backbone in standard cytotoxic treatment of early breast cancer and thus, further analyses evaluating the impact of anthracyclines for adjuvant chemotherapy in high-risk HER2-negative early breast cancer patients have to come from taxane-treated patient cohorts. We, therefore, compared the outcome of high-risk early breast cancer patients treated either with six cycles of TC or sequential anthracycline/ taxane chemotherapy using a large pooled set of individual patient data from two randomised Phase III clinical trials, PlanB and SUCCESS C. Both studies were originally designed as separate non-inferiority trials. The results of PlanB have already been published, showing that TC6 was equally effective as sequential anthracycline/taxane chemotherapy in HER2-negative early breast cancer [10]. Results of the similarly designed SUCCESS C trial have not been published before and are first presented as part of thisnot preplanned-pooled analysis, which was conducted to increase the statistical power and facilitate exploratory analyses of the effects of anthracycline-containing vs anthracycline-free chemotherapy in clinically meaningful subgroups.

\section{MATERIALS AND METHODS Study designs}

Individual patient data from the two German prospective multicenter randomised Phase III trials "PlanB" (NCT01049425, 91 centres) and "Success $C^{\prime \prime}$ (NCT00847444, 231 centres) were combined for a pooled analysis. In both trials, patients were assigned to an anthracycline-free treatment cohort (experimental arm "A-free", consisting of six cycles of TC6) or an anthracycline-containing chemotherapy arm ("A-containing"; AC-T), based on the initially administered chemotherapy regime.
For both PlanB and Success C, the experimental A-free chemotherapy arm consisted of six cycles of docetaxel $75 \mathrm{mg} / \mathrm{m}^{2}$ and cyclophosphamide $600 \mathrm{mg} / \mathrm{m}^{2}$ (TC6) every 3 weeks. The standard treatment A-containing arm in PlanB consisted of four cycles of epirubicin $90 \mathrm{mg} / \mathrm{m}^{2}$ and cyclophosphamide $600 \mathrm{mg} / \mathrm{m}^{2}$ followed by four cycles of docetaxel $100 \mathrm{mg} / \mathrm{m}^{2}$ every 3 weeks (EC-DOC). The A-containing standard treatment arm of the Success C trial comprised three cycles of 5 -fluorouracil $500 \mathrm{mg} / \mathrm{m}^{2}$, epirubicin $100 \mathrm{mg} / \mathrm{m}^{2}$ and cyclophosphamide $500 \mathrm{mg} / \mathrm{m}^{2}$ followed by three cycles of docetaxel $100 \mathrm{mg} / \mathrm{m}^{2}$ every 3 weeks (FEC-DOC) [11].

Adjuvant therapy with radiotherapy and/or endocrine treatment was allowed in both studies according to the national guideline recommendations. The primary endpoint of both studies was the comparison of DFS between both treatment arms. DFS was defined as time interval without any invasive local, regional or distant disease recurrence. Secondary endpoints included OS and safety. Toxicities were regularly assessed during treatment and afterwards. Follow-up examinations were performed according to national guidelines over at least 5 years. In both studies, various translational research programs complemented the clinical trials $[12,13]$.

\section{Patients}

Within the PlanB trial, 3198 patients were recruited between 2009 and 2011, and the Success C study enrolled 3643 patients from 2008 to 2011. All randomised patients were $\geq 18$ years and fulfilled the defined inclusion criteria. In both studies, patients with high-risk, HER2-negative invasive early breast cancer and no evidence of distant metastases were eligible after completion of local breast cancer therapy with complete resection of the tumour and axillary surgery.

In the PlanB study, the definition of high-risk patients included women with node-positive early breast cancer or node-negative tumours and additional risk factors like tumours $\geq \mathrm{T} 2$-Tumours, histopathological grade (G) $2 / 3$, high urokinase plasminogen activator/plasminogen activator inhibitor-1 (UPA/PAl-1), negative hormone-receptor (HR) status or age $\leq 35$ years. The histological type was reviewed by central pathology [14]. After an early amendment, prospective genomic testing by OncotypeDX was offered to all patients with HR + /HER2- disease with 0-3 positive lymph nodes and omission of chemotherapy were allowed with a Recurrence Score $\leq 11$. For PlanB, $n=3198$ were screened and $n=2449$ randomised. Only patients with chemotherapy treatment and available follow-up $(n=2281)$ were included in the pooled analysis. For Success $C$, high-risk early breast cancer was similarly defined as positive axillary lymph nodes or women with node-negative disease but at least pT2-Tumours or histopathological grade 3 , age $\leq 35$ or negative hormone-receptor status. All 3463 patients randomised for the Success C trial were included in the pooled analysis.

Patients with inflammatory breast cancer, severe comorbidities or relevant cardiac disorders were not eligible for both trials. All participating patients provided written informed consent. Both trials were approved by the German ethics boards and conducted in accordance to the Declaration of Helsinki.

Adverse events were assessed according to the National Cancer Institute Common Toxicity Criteria (CTCAE) version 3.0 and were collected after each cycle of chemotherapy and 3 to 4 weeks after the last chemotherapy treatment.

Biological subtypes were defined based on hormone-receptor status and histopathological grade as follows: "luminal A-like" tumours were defined as HR-positive, G1/2, "luminal B-like" tumours as hormone receptorpositive, G3, and triple-negative (TN) tumours as hormone-receptornegative, HER2-negative (HER2-negative primary tumour was a mandatory inclusion criterion in both studies).

\section{Statistical analysis}

Both studies were originally designed as non-inferiority trials, albeit with different non-inferiority margins. For PlanB, the hazard ratio based noninferiority margin was equivalent to a $4.4 \%$ difference in 5 -year DFS, while the non-inferiority margin for SUCCESS C was set to a $4.0 \%$ difference in 5 -year DFS. However, the pooled analysis was not primarily intended and there was no prospective statistical plan for the analysis of the combined dataset; thus, the analysis of the pooled data was not performed according to an inferiority trial design. The pooled analysis had a (retrospectively calculated) power of $80 \%$ to detect a $2.0 \%$ difference in 5 -year DFS, equivalent to a hazard ratio of 1.21 (two-sided test with $a=0.05$ ).

Patient outcomes in terms of DFS and OS were analysed following the standardised definitions for efficacy endpoints (STEEP) criteria [15]. 
Accordingly, DFS was defined as the time from randomisation to the earliest date of disease progression (any invasive ipsilateral, regional, contralateral, and distant disease recurrence, second primary tumours, or death from any cause; non-invasive, in-situ cancer events were excluded) or the date of censoring. OS was defined accordingly with death from any cause as an event. Survival rates based on time-to-event data were estimated by the Kaplan-Meier product-limit method and survival curves were compared using log-rank tests. Hazard ratios (HRs) with 95\% confidence intervals were estimated using univariable and multivariable Cox models adjusted for hormone-receptor (HR) status (negative, positive), histological grade $(G 1, G 2, G 3)$, age $(\leq 40,41-60,>60)$, menopausal status (premenopausal, postmenopausal), type of surgery (breast conserving, mastectomy, other), pT (pT1, pT2, pT3/pT4), pN (pN0/pN1, pN2/pN3), histological type (ductal, lobular, other), and study (Success C, PlanB). The two-way interactions between the chemotherapy treatment arm and each of the different factors presented for all of the exploratory subgroup analyses (see forest plots) were calculated using a Cox regression model with the two main effects and the corresponding two-way interaction (not adjusted for other factors). In two cases, we explored the relationships between the chemotherapy treatment arm and two of the other factors simultaneously in more detail by calculating three-way interactions (chemotherapy treatment arm * nodal status* HR status; chemotherapy treatment arm* nodal status* histological type; see "Results"). This was done to more specifically identify clinically meaningful patient subgroups that might benefit from A-containing chemotherapy in cases where the results of the two-way interactions warranted further investigations. To achieve this, we used adjusted cox regression models that included all main effects, the two two-way interactions between the chemotherapy treatment arm and both of the two other factors and the three-way interaction. We did not implement forward, backward or stepwise selection procedures and results of the full models only are reported here.

\section{RESULTS}

\section{Baseline characteristics}

For this pooled analysis, data from 5924 patients (2281 patients in PlanB and 3643 in Success C) with follow-up were available. In total, 2980 patients were randomised to TC6 and 2944 patients to AC-T (see Fig. 1 for CONSORT diagram). Median follow-up was 62.0 months (60.0 months PlanB, 64.6 months Success C). The median age was 55 years, $51.7 \%$ of patients had nodal-positive and $21.6 \%$ HR-negative disease. Baseline patient and tumour characteristics were well-balanced between the two chemotherapy treatment arms of this pooled analysis (Table 1). Detailed

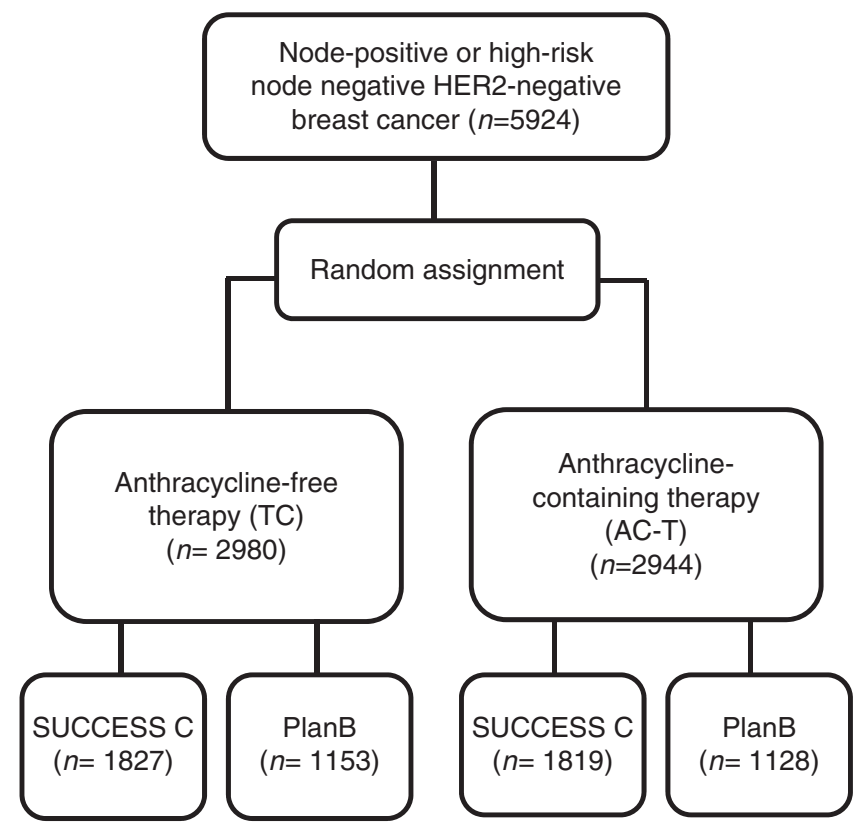

Fig. 1 Consort Diagram. CONSORT patient flow diagram of the PlanB and Success C pooled analysis. information on patient and tumour characteristics according to trial (Success C, PlanB) is provided in Supplementary Table S1.

\section{Outcome}

DFS of patients receiving A-free vs A-containing chemotherapy was quite similar in univariable analysis (hazard ratio, $\mathrm{HR}=1.05$; 95\% confidence interval, $\mathrm{Cl}: 0.89-1.24, P=0.57$; Fig. $2 \mathrm{a}$ ) and in multivariable analysis ( $\mathrm{HR}=1.01,95 \% \mathrm{Cl}$ : $0.86-1.19, P=0.91$; see Supplementary Table S2). Five-year DFS rate was $90.0 \%$ (283 events) in the A-containing and $89.3 \%$ (298 events) in the A-free group (see Supplementary Table S3 for a detailed list of DFS events). Similarly, OS did not differ between the A-free vs A-containing study arms in univariable $(\mathrm{HR}=1.00$; $95 \%$ confidence interval, $\mathrm{Cl}$ : $0.80-1.26, P=1.00$; Fig. $2 \mathrm{~b}$ ) and adjusted multivariable analysis ( $\mathrm{HR}=0.97,95 \% \mathrm{Cl}$ : $0.77-1.22, P=0.79)$; 5 -year OS rates were $94.9 \%$ (149 events) and 95.0\% (149 events) in the A-free and A-containing treatment arms, respectively (Fig. 2b).

The forest plots for the comparison between patients receiving anthracycline-free or anthracycline-containing chemotherapy for all subgroups tested including the $P$ values for the two-way interactions are shown in Fig. 3 for DFS (details on OS are presented in the supplement). There were no significant differences in DFS between A-free and A-containing chemotherapy in any of the three different biological tumour subtypes (luminal A-like: $\mathrm{HR}=1.09,95 \% \mathrm{Cl}: 0.83-1.42, P=0.55$; luminal $\mathrm{B}$ like: $\mathrm{HR}=1.07,95 \% \mathrm{Cl}: 0.78-1.48, P=0.68$; triple-negative: $\mathrm{HR}=$ $0.99,95 \% \mathrm{Cl}: 0.76-1.30, P=0.96)$. Similar results with no differences between A-free and A-containing chemotherapy were obtained for OS (luminal A-like: $\mathrm{HR}=0.94,95 \% \mathrm{Cl}$ : 0.64-1.39, $P=$ 0.76; luminal B-like: $\mathrm{HR}=0.92,95 \% \mathrm{Cl}$ : $0.57-1.48, P=0.72$; triplenegative: $\mathrm{HR}=1.08,95 \% \mathrm{Cl}: 0.76-1.53, P=0.66)$. Furthermore, there were no significant two-way interactions between chemotherapy treatment arm (A-free or A-containing) and biological subtype for DFS $(P=0.896)$ or OS $(P=0.828)$, indicating that there is no evidence for a survival benefit of either A-containing or A-free chemotherapy depending on biological subtype. Similar results with no significant two-way interactions with respect to both DFS and OS were obtained with regard to subgroups defined by study (PlanB, Success C), menopausal status (premenopausal, postmenopausal), age ( $\leq 40$ years, $41-60$ years, $>60$ years), tumour size (pT1, pT2, pT3/pT4), HR status (negative, positive), and histological grade (G1, G2, G3).

No difference in DFS between the two chemotherapy arms was found for patients with pN0/pN1 tumours $(\mathrm{HR}=0.95,95 \% \mathrm{Cl}$ : $0.79-1.15, P=0.61$; Supplementary Fig. S1A). In contrast, a significant difference between TC6 and AC-T in the subgroup analyses was found for patients with more than three affected lymph nodes (pN2/pN3 tumours), who had a significantly worse DFS when treated with A-free chemotherapy $(\mathrm{HR}=1.48,95 \% \mathrm{Cl}$ : 1.04-2.13, $P=0.031$; Supplementary Fig. S1B). The resulting significant two-way interaction $(P=0.033)$ between chemotherapy arm (TC6 or AC-T) and nodal status (pN0/pN1 or pN2/pN3) suggests that the effect of AC-T depends on the extent of lymph node involvement, with patients that have pN2 or pN3 tumours benefitting from A-containing chemotherapy. Further analyses showed that the two-way interaction between chemotherapy arm (TC6 or AC-T) and nodal status (pN0/pN1 or pN2/pN3) was significant in HR-positive tumours $(P=0.019)$ but not in triplenegative tumours $(P=0.89)$, indicating that the benefit from A-containing chemotherapy in patients with $\mathrm{pN} 2 / \mathrm{pN} 3$ tumours seems to be restricted to luminal-like tumours only. To formally test whether the effect of nodal status on the efficacy of AC-T depends on hormone-receptor status, we performed an adjusted multivariable cox regression model (including all main effects, the two-way interactions between chemotherapy regime and nodal status and between chemotherapy regime and hormone-receptor status, and the three-way interaction between chemotherapy arm, nodal status and hormone receptor status). However, this model 
Table 1. Baseline and clinic-pathological characteristics of patients according to chemotherapy arm.

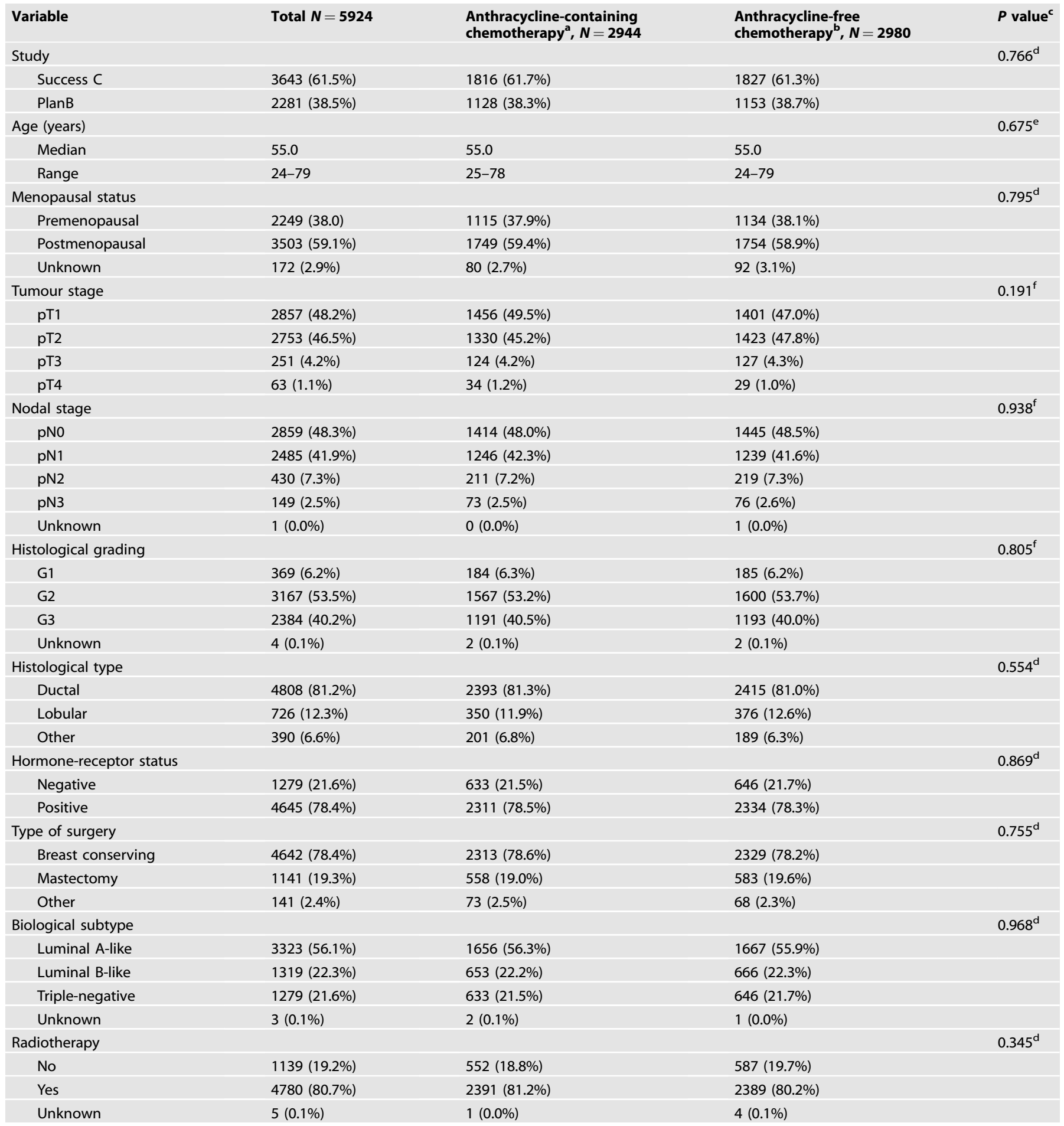

asuccess C: FEC-Doc; $3 \times$ fluorouracil $_{500}$-epirubicin $_{100^{-}}$-cyclophosphamide $_{500} \mathrm{q} 3 \mathrm{~W}$ followed by $3 \times$ docetaxel $_{100}$ q3W; PlanB: EC-Doc; $4 \times$ epirubicin $_{90^{-}}$ cyclophosphamide $_{600} \mathrm{q} 3 \mathrm{w}$ followed by $4 \times$ docetaxel $_{100} \mathrm{q} 3 \mathrm{w}$.

buccess C and PlanB: Doc-C; $6 \times$ docetaxel $_{75}$-cyclophosphamide ${ }_{600}$ q3w.

${ }^{\mathrm{C}}$ All tests without unknowns.

${ }^{\mathrm{d}}$ Chi-square test.

${ }^{\mathrm{C}}$ Mann-Whitney $U$ test.

${ }^{f}$ Cochran-Armitage test for trend. 

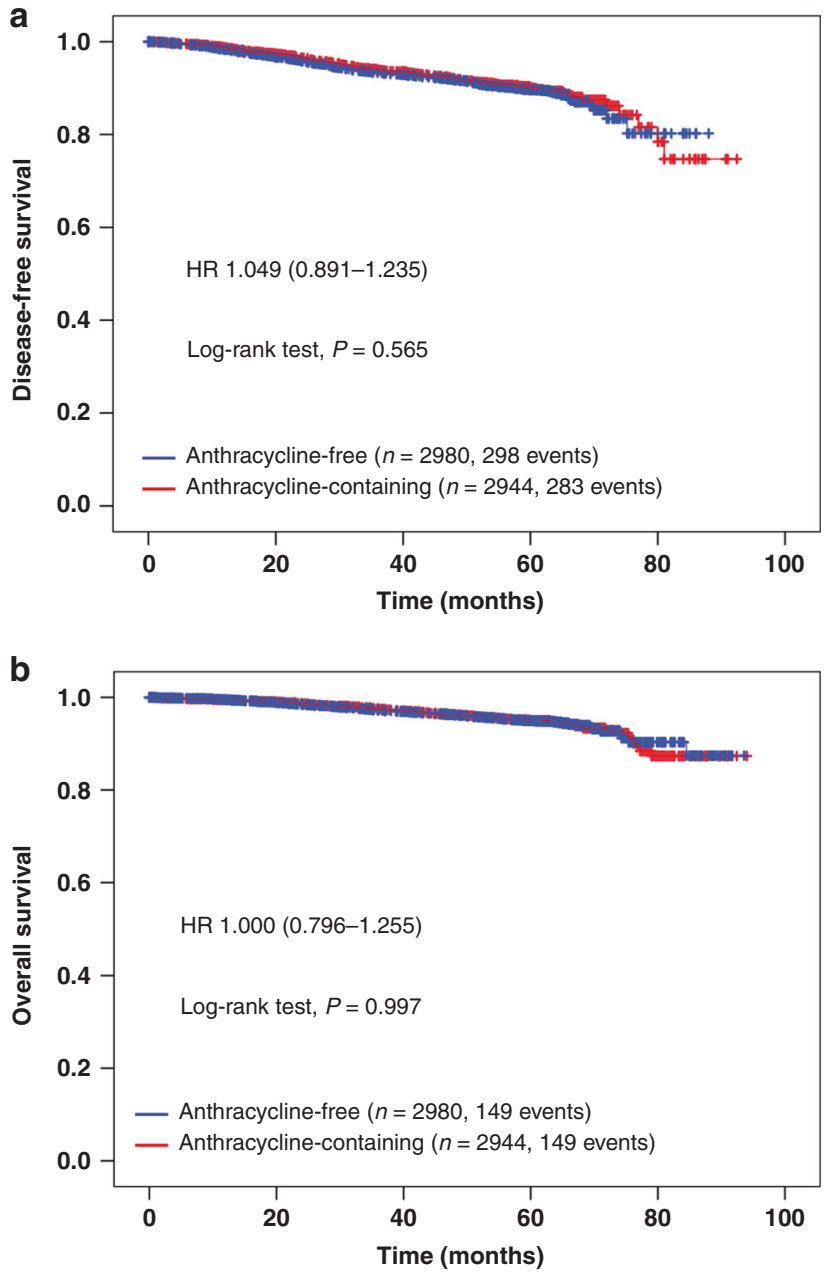

Fig. 2 Kaplan-Meier plots showing outcome for anthracycline-free vs. anthracycline-containing chemotherapy. Kaplan-Meier plots for disease-free survival (a) and overall survival (b) according to chemotherapy arm (anthracycline-free vs. anthracycline-containing) of the PlanB and Success C trials (data pooled).

revealed no significant three-way interaction between chemotherapy arm, nodal status and hormone-receptor status $(P=0.245)$, indicating that the influence of nodal status on the efficacy of $A C$ $\mathrm{T}$ was not significantly modulated by hormone-receptor status.

The only other significant difference between A-free and A-containing chemotherapy was obtained in the subgroup analysis for lobular cancer. Patients with lobular carcinomas receiving TC6 had significantly worse DFS compared to patients with lobular carcinomas receiving A-containing chemotherapy ( $\mathrm{HR}=2.07,95 \% \mathrm{Cl}: 1.20-3.57, P=0.008$; Supplementary Fig. S2B). No difference in DFS between the two chemotherapy regimens was found for patients with ductal carcinomas ( $\mathrm{HR}=0.97,95 \% \mathrm{Cl}$ : $0.81-1.16, P=0.75$; Supplementary Fig. S2A) or patients with carcinomas of a histological type other than ductal or lobular ( $\mathrm{HR}=1.01,95 \% \mathrm{Cl}: 0.56-1.81, P=0.97$; Supplementary Fig. S2C). Furthermore, the two-way interaction between chemotherapy treatment arm and histological type was significant $(P=0.041)$, indicating that patients with lobular carcinomas-in contrast to patients with other tumour histology-show better DFS with A-containing chemotherapy.

We also performed an exploratory analysis to investigate whether the effect of anthracycline-containing chemotherapy observed in patients with lobular carcinomas was dependent on menopausal status. However, there was no significant two-way interaction between chemotherapy treatment arm and menopausal status $(P=0.091)$, indicating that the benefit of anthracyclinecontaining chemotherapy in patients with lobular carcinomas was not affected by menopausal status. More details and results of this exploratory analysis are presented in the Supplementary Material (Supplementary Material S1).

Taken together, our data (i.e. the significant two-way interactions) suggest that both patients with lobular tumours and patients with $\mathrm{pN} 2 / \mathrm{pN} 3$ tumours might benefit from A-containing chemotherapy in terms of longer DFS. To explore these effects in more detail, we used an adjusted multivariable cox regression model including all main effects, the two-way interactions between chemotherapy treatment arm and both nodal status and histological type, and the three-way interaction between chemotherapy arm, nodal status and histological type. In this model, the two-way interactions between chemotherapy treatment $\mathrm{arm}^{*}$ nodal status and chemotherapy treatment arm* histological type became non-significant $(P=0.688$ and $P=0.746$, respectively), while the three-way interaction was significant $(P=$ 0.028; see Supplementary Table S4). This result indicates that the benefit of A-containing chemotherapy was not evident in all patients with pN2/pN3 tumours and in all patients with lobular carcinomas, but was only seen in patients that had both, i.e. a lobular tumour with more than three affected lymph nodes (HR $3.52,95 \% \mathrm{Cl}$ : $1.43-8.65$; Fig. 4 a), with a 5 -year DFS rate of $89.0 \%$ in the A-containing and $65.8 \%$ in the A-free group. In contrast, there were no significant differences in DFS between A-containing and A-free chemotherapy for patients with pN0/pN1 lobular carcinomas (HR 1.30, 95\% Cl: 0.63-2.68; Fig. 4b), for patients with pN2/ pN3 ductal carcinomas (HR 1.16, 95\% Cl: 0.76-1.76; Fig. 4c) and for patients with $\mathrm{pN} 2 / \mathrm{pN} 3$ carcinomas with histological types other than ductal or lobular (HR 1.35, 95\% Cl: 0.25-7.37, Fig. 4d).

There were no significant two-way interactions between the chemotherapy arm (anthracycline-free or anthracycline-containing) and any of the subgroup variables tested with regard to OS (see Supplementary Fig. S3). However, similar to DFS, an adjusted multivariable cox regression model including all main effects, the two-way interactions between chemotherapy treatment arm and both nodal status and histological type, and the three-way interaction between chemotherapy arm, nodal status and histological type yielded a significant three-way interaction $(P=$ 0.022 ), confirming an OS benefit for patients with lobular pN2/pN3 tumours (univariable HR $3.41,95 \% \mathrm{Cl}$ : $1.10-10.56, P=0.034$; see Supplementary Fig. S4).

\section{Adverse events}

Table 2 lists the toxicity summary from PlanB and Success $C$ with the description of well-known adverse events. During the course of the Success C study, 24 unexpected serious events (SUSARs) were observed; one SUSAR was associated with lethal outcome (hepatic insufficiency, Doc-C arm). In PlanB, six treatment-related deaths were reported [10]. Overall, grade $3 / 4$ adverse events were observed significantly more often in patients receiving anthracycline-containing chemotherapy than those receiving anthracycline-free chemotherapy $(76.3 \%$ vs. $70.1 \% ; P<0.001)$. More specifically, patients in the anthracycline-containing chemotherapy arm had significantly more leukopenia grade 3/4, nausea grade $3 / 4$, fatigue grade $3 / 4$, vomiting grade $3 / 4$ and stomatitis grade $3 / 4$ than patients in the anthracycline-free chemotherapy arm (see Table 2A). In contrast, there was no specific adverse event that occurred significantly more often in the anthracycline-free chemotherapy arm. The frequency of antibiotic treatment did not differ significantly between the two chemotherapy arms, but G-CSF treatment was applied significantly more often in the anthracycline-containing chemotherapy arm (Table 2B). 


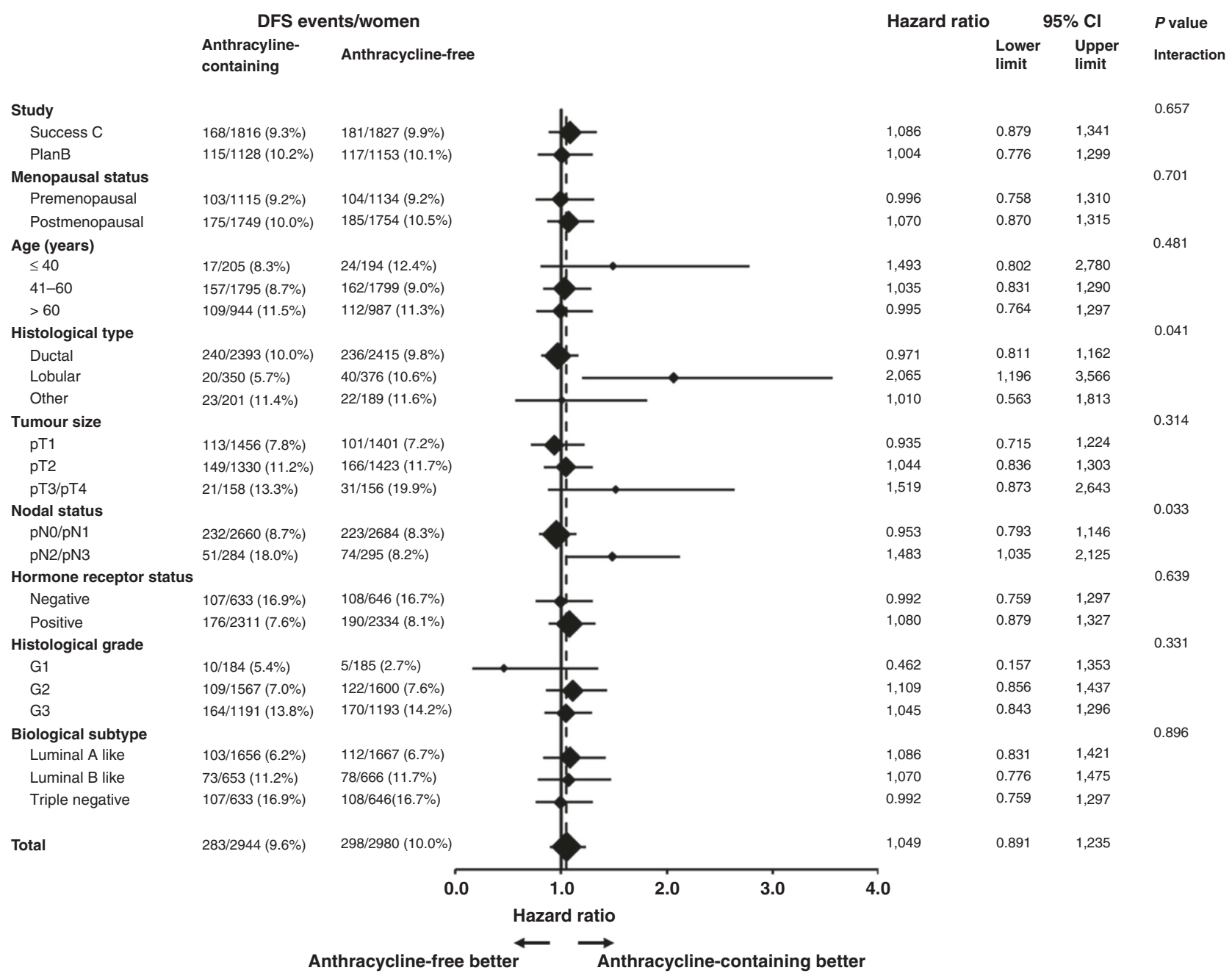

Fig. 3 Forest plot showing disease-free survival with anthracycline-free or anthracycline-containing chemotherapy according to the different patient and tumour characteristic subgroups. Forest plot showing results of explorative subgroup analyses in terms of the comparison of disease-free survival between patients with anthracycline-free or anthracycline-containing chemotherapy according to the different patient and tumour characteristic subgroups. The diamonds indicate the hazard ratios (anthracycline-free vs. anthracyclinecontaining chemotherapy), and diamond size is proportional to the number of patients per subgroup. The horizontal lines indicate the corresponding $95 \%$ confidence intervals for the hazard ratios. The solid vertical line represents a hazard ratio of 1.0 (i.e., no difference in survival between anthracycline-free or anthracycline-containing chemotherapy), and the dashed vertical line represents the hazard ratio for the overall analysis with all 5924 patients (hazard ratio 1.049).

\section{DISCUSSION}

Based on the results of our pooled analysis with individual data from almost 6000 patients, we found that six cycles of TC (TC6) provide similar efficacy compared to an anthracycline-containing regimen in most patients with HER2-negative early breast cancer and showed significantly lower incidence of overall grade 3/4 toxicities. However, subgroup analyses indicate that patients with a high tumour burden in terms of four or more involved lymph nodes and patients with lobular tumours might benefit from anthracycline-containing chemotherapy, at least in terms of increased DFS. A more detailed analysis incorporating two-way and three-way interaction tests showed that this benefit may be limited to the subset of patients with pN2/3 lobular tumours only. These findings are in contrast to the published analysis by Blum et al. from the ABC trials ( $n=4242$ patients), which could not demonstrate non-inferiority of anthracycline-free therapy (TC6) compared to anthracycline-containing regimens with regard to invasive DFS in patients with high-risk HER2-negative early breast cancer [9]. The three adjuvant trials included (US Oncology Research (USOR) 06-090, National Surgical Adjuvant Breast and Bowel Project (NSABP) B-46-I/USOR 07132 and NSABP B-49) mostly used concomitant taxanes (docetaxel, doxorubicin and cyclophosphamide-TAC chemotherapy regime); only in the NSABP B-49 trial investigators could choose both between concomitant or sequential taxanes and between paclitaxel or docetaxel.

The impact of different available agents (docetaxel, paclitaxel, nab-paclitaxel) and taxane schedules (concomitant or sequential) within anthracycline/taxane-chemotherapies on outcome in early breast cancer patients has been controversially discussed. DFS in high-risk breast cancer patients, treated with anthracyclinecontaining chemotherapy, is improved with weekly paclitaxel (odds ratio (OR) for DFS 1.27; $P=0.006$ ) and also docetaxel every 3 weeks (OR 1.23; $P=0.02$ ) compared to paclitaxel every 3 weeks [18]. With regard to taxane schedules, two large trials revealed no difference in efficacy between concomitant versus sequential anthracycline/taxane-based chemotherapy regimens $[16,17]$. In contrast, a study from Swain et al. from 2010 showed a DFS benefit with sequential AC-T compared to doxorubicin-docetaxel (AT) or concurrent AC-T and, in addition, also an improved OS with sequential AC-T compared to AT [18]. Similarly, Oakmen et al. found an improvement of OS after 8 years in node-positive early breast cancer patients treated with sequential docetaxel 

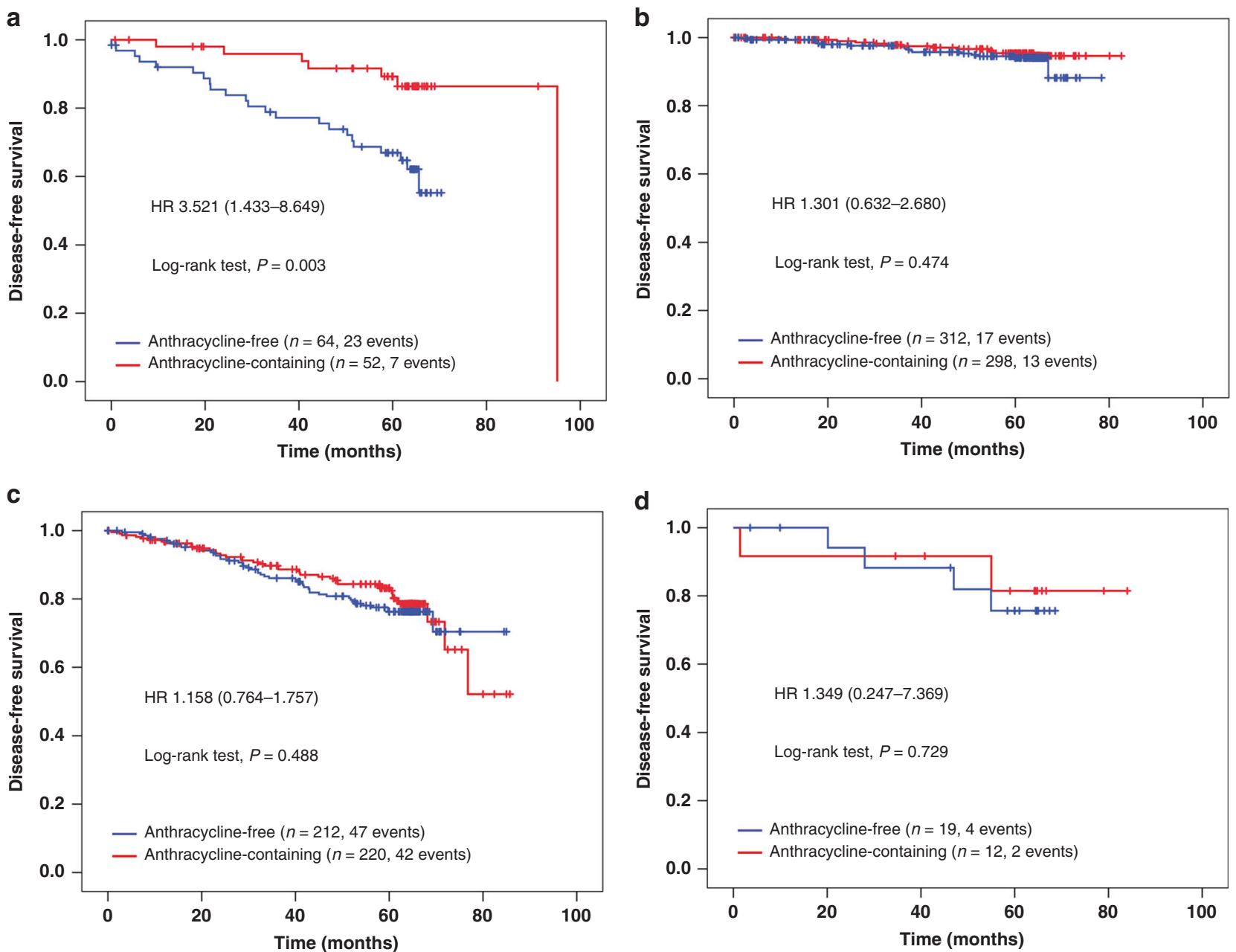

Fig. 4 Kaplan-Meier showing disease-free survival in different subgroups according to chemotherapy arm. Kaplan-Meier plots for diseasefree survival in different subgroups according to chemotherapy arm (anthracycline-free vs. anthracycline-containing) of the PlanB and Success $\mathrm{C}$ trials (data pooled) for patients with pN2/pN3 lobular tumours (a), pN0/pN1 lobular tumours (b), pN2/pN3 ductal tumours (c), and pN2/pN3 tumours with another histological type (d). In addition to the results of the log-rank test, univariable hazard ratios and $95 \%$ confidence intervals are shown.

compared to concurrent doxorubicin-docetaxel [19]. A metaanalysis of three Phase III trials likewise showed a significant improvement of DFS and OS with sequential versus concurrent anthracycline/taxane-containing chemotherapy in adjuvant treatment of early breast cancer patients (DFS: RR $0.90,95 \% \mathrm{Cl}$ : $0.84-0.98, P=0.01$; OS: RR 0.88, 95\% Cl: $0.79-0.98, P=0.02$ ) [20]. These findings indicating a survival benefit of sequential versus concurrent anthracycline/taxane-containing chemotherapy might be related to the fact that the cumulative dose of taxanes usually is higher for sequential than simultaneous taxane application within an anthracycline/taxane-chemotherapy regime. In addition, the use of sequential taxanes in anthracycline/taxane chemotherapy often leads to prolonged treatment duration which might also influence treatment efficacy.

These points could explain the discordant conclusion of PlanB/ Success $C$ compared to the $A B C$ trials where mostly TAC with simultaneous taxanes was used as a chemotherapy regime. In addition, median follow-up time in the $A B C$ trial was 3.3 years with only 2.2 years in the largest of the three included trials (NSABP B49) compared to 62 months (5.16 years) in our pooled analysis. Longer follow-up is especially important in $\mathrm{HR}+$ disease, given the frequent occurrence of late recurrences (after 5 years) in this subset of early breast cancer patients [21]. In addition, no benefit for any chemotherapy regime in terms of increased iDFS was seen in the subset of patients with pNO and the patients treated within the largest trial NSABP B-49 $(n=1819)$.

Though the used taxane dose per cycle is lower (but cumulative taxane dose is somewhat higher) in the anthracycline-free TC6 regime (docetaxel $75 \mathrm{mg} / \mathrm{m}^{2}$ every three weeks) compared to anthracycline-containing regimes with sequential taxane use (docetaxel $100 \mathrm{mg} / \mathrm{m}^{2}$ every three weeks), TC6 showed comparable efficacy in the included patients. However, according to our results, especially the subset of patients with pN2/pN3 lobular tumours seems to profit from anthracycline-containing chemotherapy. This particular subgroup showed not only a clearly improved DFS but also better OS with anthracyclines. While there is evidence that high-risk lobular breast cancers clearly benefit from chemotherapy [22], detailed knowledge regarding particular sensitivity to anthracycline-containing vs anthracycline-free chemotherapy in different histological breast cancer subtypes is currently not available. Several markers have been postulated to indicate sensitivity to anthracycline-containing chemotherapy including topoisomerase II alpha (TOP2A) as the most promising one, but-as pointed out by a recent review-results of retrospective analyses are mostly inconsistent and difficult to interpret, and confirmation from prospectively randomised studies that TOP2A amplification is associated with increased sensitivity to anthracyclines is still lacking [23]. 
Table 2. (A) Frequency (number of patients) of grade $3 / 4$ adverse events (overall, most common adverse events; CTCAE V 3.0) according to chemotherapy arm (anthracycline-containing vs. anthracyclinefree); (B) antibiotic treatment and G-CSF treatment according to chemotherapy arm (anthracycline-containing vs. anthracycline-free).

\begin{tabular}{|c|c|c|c|}
\hline Variable & $\begin{array}{l}\text { Anthracycline- } \\
\text { containing } \\
\text { chemotherapy } \\
\text { (FEC-Doc/EC- } \\
\text { Doc }^{\text {b } N=2944)}\end{array}$ & $\begin{array}{l}\text { Anthracycline-free } \\
\text { chemotherapy } \\
\text { (Doc-C'; } N=2980 \text { ) }\end{array}$ & $P$ value \\
\hline \multicolumn{4}{|l|}{ (A) } \\
\hline $\begin{array}{l}\text { Any adverse } \\
\text { event }\end{array}$ & 2245 (76.3\%) & 2089 (70.1\%) & $<0.001^{*}$ \\
\hline Anaemia & $20(0.7 \%)$ & $21(0.7 \%)$ & 0.91 \\
\hline Leukopenia & 1509 (51.3\%) & $1358(45.6 \%)$ & $<0.001^{*}$ \\
\hline Neutropenia & 1187 (40.3\%) & 1101 (36.9\%) & 0.008 \\
\hline Nausea & $88(3.0 \%)$ & $40(1.3 \%)$ & $<0.001^{*}$ \\
\hline Fatigue & 131 (4.4\%) & $83(2.8 \%)$ & $0.001^{*}$ \\
\hline Vomitting & $53(1.8 \%)$ & $18(0.6 \%)$ & $<0.001^{*}$ \\
\hline Stomatitis & 57 (1.9\%) & $26(0.9 \%)$ & $<0.001^{*}$ \\
\hline Constipation & $21(0.7 \%)$ & $12(0.4 \%)$ & 0.11 \\
\hline Diarrhoea & 55 (1.9\%) & $63(2.1 \%)$ & 0.50 \\
\hline SGPT elevation & $46(1.6 \%)$ & 39 (1.3\%) & 0.41 \\
\hline $\begin{array}{l}\text { SGOT } \\
\text { elevation }\end{array}$ & $10(0.3 \%)$ & $6(0.2 \%)$ & 0.31 \\
\hline Pain & $68(2.3 \%)$ & 45 (1.5\%) & 0.024 \\
\hline Infection & $59(2.0 \%)$ & $78(2.6 \%)$ & 0.12 \\
\hline Neuropathy & 45 (1.5\%) & $23(0.8 \%)$ & 0.006 \\
\hline Arthralgia & 45 (1.5\%) & $29(1.0 \%)$ & 0.054 \\
\hline $\begin{array}{l}\text { Febrile } \\
\text { neutropenia }\end{array}$ & 114 (3.9\%) & 145 (4.9\%) & 0.062 \\
\hline \multicolumn{4}{|l|}{ (B) } \\
\hline $\begin{array}{l}\text { Antibiotic } \\
\text { treatment }\end{array}$ & & & 0.16 \\
\hline No & $2217(75.3 \%)$ & $2288(76.8 \%)$ & \\
\hline Yes & $726(24.7 \%)$ & $688(23.1 \%)$ & \\
\hline Unknown & $1(0.0 \%)$ & $4(0.1 \%)$ & \\
\hline $\begin{array}{l}\text { G-CSF } \\
\text { treatment }\end{array}$ & & & $0.001^{*}$ \\
\hline No & 1598 (54.3\%) & 1734 (58.2\%) & \\
\hline Yes & $1285(43.6 \%)$ & 1162 (39.0\%) & \\
\hline Unknown & $61(2.1 \%)$ & $84(2.8 \%)$ & \\
\hline
\end{tabular}

Data pooled from the Success $C$ and PlanB trials.

${ }^{\text {aFEC-Doc: } 3 \times \text { fluorouracil }}{ }_{500}$-epirubicin ${ }_{100}$-cyclophosphamide ${ }_{500}$ q3W followed by $3 \times$ docetaxel $_{100} \mathrm{q} 3 \mathrm{w}$.

${ }^{b}$ EC-Doc: $4 \times$ epirubicin $_{90}$-cyclophosphamide 600 q3w followed by $4 \times$ docetaxel $_{100} \mathrm{q} 3 \mathrm{w}$.

${ }^{c}$ Doc-C: $6 \times$ docetaxel $_{75}$-cyclophosphamide ${ }_{600}$ q3w.

${ }^{\mathrm{d}}$ Chi-square test.

*Significant after significance level was adjusted for multiple comparisons using Bonferroni correction.

A significant two-way interaction between chemotherapy arm and nodal stage for patients with HR-positive disease was reported by Blum et al. [9]. Whereas the analysis of Blum et al. also suggested potential benefits of A-containing chemotherapy for patients with HR-negative tumours (triple-negative) [24], our data do not support this finding. Based on our results, patients with triple-negative tumours do not seem to benefit from additional treatment with anthracyclines. Results from the neoadjuvant setting suggest that they may gain further benefit by the addition of platinum to anthracycline-taxane chemotherapy $[25,26]$. Nevertheless, the anthracycline-free combination of docetaxel and carboplatin has also shown high efficacy in neoadjuvant triple-negative breast cancer [27] which was even comparable to that of an anthracycline-taxane and platinum sequence in the NeoSTOP trial [28]. Recent data from an adjuvant Phase III trial in triple-negative early breast cancer even showed improved DFS of six cycles carboplatin-paclitaxel compared to $3 \times$ FEC and $3 \times$ DOC [29]. In addition, our results indicate that anthracycline-free chemotherapy is also a valid option for younger patients ( $<40$ years) or patients with larger tumours (pT3/pT4).

The restriction of "taxane use" in the A-containing treatment arm to sequential docetaxel $100 \mathrm{mg} / \mathrm{m}^{2}$ every 3 weeks for three or four cycles only is a major strength of the presented pooled analysis and prevents potential bias through different taxane regimes, agents and application intervals. However, a limiting factor is that the standard treatment arm of this pooled analysis includes two different AC-T chemotherapy regimens with different duration of chemotherapy exposure consisting of either $3 \times \mathrm{FEC} /$ $3 \times \mathrm{DOC}$ (Success C) or $4 \times \mathrm{EC} / 4 \times \mathrm{DOC}$ (PlanB). In addition, though FEC-DOC is an effective regimen, 5-FU is not considered standard anymore in this setting, as the addition of 5-FU to an anthracycline/cyclophosphamide combination failed to show any relevant benefit in improvement of DFS [30]. However, multivariable testing was adjusted for the different treatment regimens and thereby, the interaction results are not biased by a "trialeffect". Another limitation is the fact that-despite the large overall sample size of this pooled analysis-there is still a $20 \%$ risk to show a false negative result with regard to the detection of a $2 \%$ difference in 5-year DFS (as the retrospectively calculated power was $80 \%$ ), and that some of the subgroups analysed were still rather small with a corresponding further loss of statistical power for the post hoc comparisons involving these subgroups. Lastly, median follow-up in our pooled analysis comprises only 5 years and thus late disease recurrences might have been missed.

Limiting the incorporation of anthracyclines only for selected patients is desirable to prevent avoidable toxicities without a gain in efficacy. Our study represents currently the largest for assessing the impact of the addition of anthracyclines to a taxane-based regimen in intermediate to high-risk HER2-negative early breast cancer. The presented pooled analysis clearly addresses the question of TC vs AC-T therapy in adjuvant breast cancer treatment. The data suggest that six cycles of TC represent an effective chemotherapy option with less toxicity for HER2-negative early breast cancer and based on these results the indication for TC6 could be expanded towards intermediate-risk early breast cancer patients. Exploratory subgroup analyses indicated that some high-risk patients may benefit from anthracyclinecontaining chemotherapy in terms of improved DFS but not OS. Further evaluation of those results by additional interaction analyses showed no benefit for the majority of patients. Only the subgroup of lobular-invasive pN2/pN3 early breast cancerderived significant benefit regarding better DFS and OS with the addition of anthracycline. Thus, a clear benefit of anthracyclinecontaining chemotherapy is observed only for about $2 \%$ of the overall HER2-negative early breast cancer study population in the here presented analysis (acknowledging that in the rest of the population this pooled analysis has only $80 \%$ power to detect a $2 \%$ difference in 5-year DFS). Suggesting that this specific subgroup may have different biological features, we need further information for a better understanding of this subset of patients. Our results indicate that the simple classification of patient groups only according to isolated factors like "node-positive" or "histological type" could be insufficient to identify patient subgroups that might truly benefit from anthracyclines. The impact of anthracyclines has to be addressed in future 
randomised trials specifically aimed at selected high-risk breast cancer patients.

\section{DATA AVAILABILITY}

De-identified data will be made available to other researchers, subject to the approval of a formal data access request that includes a detailed description of the purpose/scientific rationale of the proposed project. Requests are reviewed by the study groups Steering Committees and will be approved if the proposed projects have a sound scientific or patient benefit rationale. Data recipients are required to sign a formal data sharing agreement that describes the conditions for release and requirements for data transfer, storage, archiving, publication and intellectual property. Data will be available beginning 9 months and ending 5 years following article publication. Additional documents (e.g., full study protocol, informed consent form) are available on request.

\section{REFERENCES}

1. Denduluri N, Somerfield MR, Eisen A, Holloway JN, Hurria A, King TA, et al. Selection of optimal adjuvant chemotherapy regimens for human epidermal growth factor receptor 2 (HER2)-negative and adjuvant targeted therapy for HER2-positive breast cancers: an American Society of Clinical Oncology Guideline Adaptation of the Cancer Care Ontario Clinical Practice Guideline. J Clin Oncol. 2016;34:2416-27.

2. Henderson IC, Berry DA, Demetri GD, Cirrincione CT, Goldstein LJ, Martino S, et al. Improved outcomes from adding sequential paclitaxel but not from escalating doxorubicin dose in an adjuvant chemotherapy regimen for patients with nodepositive primary breast cancer. J Clin Oncol. 2003;21:976-83.

3. Roché $H$, Fumoleau $P$, Spielmann $M$, Canon J-L, Delozier $T$, Serin $D$, et al Sequential adjuvant epirubicin-based and docetaxel chemotherapy for nodepositive breast cancer patients: the FNCLCC PACS 01 trial. J Clin Oncol. 2006;24:5664-71.

4. Early Breast Cancer Trialists' Collaborative Group (EBCTCG) EBCTCG, Peto R, Davies C, Godwin J, Gray R, Pan HC, et al. Comparisons between different polychemotherapy regimens for early breast cancer: meta-analyses of longterm outcome among 100,000 women in 123 randomised trials. Lancet. 2012;379:432-44.

5. Jones SE, Savin MA, Holmes FA, O'Shaughnessy JA, Blum JL, Vukelja S, et al. Phase III trial comparing doxorubicin plus cyclophosphamide with docetaxel plus cyclophosphamide as adjuvant therapy for operable breast cancer. J Clin Oncol. 2006;24:5381-7.

6. Jones S, Holmes FA, O'Shaughnessy J, Blum JL, Vukelja SJ, Mclntyre KJ, et al. Docetaxel with cyclophosphamide is associated with an overall survival benefit compared with doxorubicin and cyclophosphamide: 7-year follow-up of US oncology research trial 9735. J Clin Oncol. 2009;27:1177-83.

7. Azim JA, de Azambuja E, Colozza M, Bines J, Piccart MJ. Long-term toxic effects of adjuvant chemotherapy in breast cancer. Annal Oncol. 2011;22:1939-47.

8. Henriksen PA. Anthracycline cardiotoxicity: an update on mechanisms, monitoring and prevention. Heart. 2018;104:971-77.

9. Blum JL, Flynn PJ, Yothers G, Asmar L, Geyer CE, Jacobs SA, et al. Anthracyclines in early breast cancer: the ABC trials-USOR 06-090, NSABP B-46-I/USOR 07132, and NSABP B-49 (NRG Oncology). J Clin Oncol. 2017;35:2647-55.

10. Nitz U, Gluz $O$, Clemens M, Malter W, Reimer T, Nuding B, et al. West German study PlanB trial: adjuvant four cycles of epirubicin and cyclophosphamide plus docetaxel versus six cycles of docetaxel and cyclophosphamide in HER2-negative early breast cancer. J Clin Oncol. 2019;37:799-808.

11. Rack B, Andergassen U, Neugebauer J, Salmen J, Hepp P, Sommer H, et al. The German SUCCESS C Study-the first European lifestyle study on breast cancer. Breast Care. 2010;5:395-400.

12. Gluz O, Nitz UA, Christgen M, Kates RE, Shak S, Clemens M, et al. West German Study Group Phase III PlanB Trial: first prospective outcome data for the 21-gene recurrence score assay and concordance of prognostic markers by central and local pathology assessment. J Clin Oncol. 2016;34:2341-9.

13. Schramm A, Schochter F, Friedl TWP, de Gregorio N, Andergassen U, AlunniFabbroni $M$, et al. Prevalence of circulating tumor cells after adjuvant chemotherapy with or without anthracyclines in patients with HER2-negative, hormone receptor-positive early breast cancer. Clin Breast Cancer. 2017;17:279-85.

14. Christgen M, Gluz O, Harbeck N, Kates RE, Raap M, Christgen $H$, et al. Differential impact of prognostic parameters in hormone receptor-positive lobular breast cancer. Cancer. 2020;126:4847-58.

15. Hudis CA, Barlow WE, Costantino JP, Gray RJ, Pritchard KI, Chapman JAW, et al. Proposal for standardized definitions for efficacy end points in adjuvant breast cancer trials: The STEEP system. J Clin Oncol. 2007;25:2127-32.

16. Swain SM, Tang G, Geyer CE, Rastogi P, Atkins JN, Donnellan PP, et al. Definitive results of a phase iii adjuvant trial comparing three chemotherapy regimens in women with operable, node-positive breast cancer: the NSABP B-38 trial. J Clin Oncol. 2013;31:3197-204.

17. Mackey JR, Pieńkowski T, Crown J, Sadeghi S, Martin M, Chan A, et al. Long-term outcomes after adjuvant treatment of sequential versus combination docetaxel with doxorubicin and cyclophosphamide in node-positive breast cancer: BCIRG005 randomized trial. Ann Oncol. 2016;27:1041-7.

18. Swain SM, Jeong J-H, Geyer CE, Costantino JP, Pajon ER, Fehrenbacher L, et al. Longer therapy, iatrogenic amenorrhea, and survival in early breast cancer. $\mathrm{N}$. Engl J Med. 2010;362:2053-65.

19. Oakman C, Francis PA, Crown J, Quinaux E, Buyse M, De Azambuja E, et al. Overall survival benefit for sequential doxorubicin-docetaxel compared with concurrent doxorubicin and docetaxel in node-positive breast cancer-8-year results of the Breast International Group 02-98 phase III trialt. Ann Oncol. 2013;24:1203-11.

20. Shao N, Wang S, Yao C, Xu X, Zhang Y, Zhang Y, et al. Sequential versus concurrent anthracyclines and taxanes as adjuvant chemotherapy of early breast cancer: a meta-analysis of phase III randomized control trials. Breast. 2012;21: 389-93.

21. Saphner T, Tormey DC, Gray R. Annual hazard rates of recurrence for breast cancer after primary therapy. J Clin Oncol. 1996;14:2738-46.

22. de Nonneville A, Jauffret C, Gonçalves A, Classe JM, Cohen M, Reyal F, et al. Adjuvant chemotherapy in lobular carcinoma of the breast: a clinicopathological score identifies high-risk patient with survival benefit. Breast Cancer Res Treat. 2019;175:379-87.

23. Hurvitz SA, McAndrew NP, Bardia A, Press MF, Pegram M, Crown JP, et al. A careful reassessment of anthracycline use in curable breast cancer. npj Breast Cancer. 2021;7:1-25.

24. Blum JL, Flynn PJ, Yothers G, Asmar L, Geyer CE, Jacobs SA, et al. Anthracyclines in early breast cancer: the ABC trials-USOR 06-090, NSABP B-46-I/USOR 07132, and NSABP B-49 (NRG Oncology). J Clin Oncol. 2017;35:2647-55.

25. von Minckwitz G, Schneeweiss A, Loibl S, Salat C, Denkert C, Rezai M, et al. Neoadjuvant carboplatin in patients with triple-negative and HER2-positive early breast cancer (GeparSixto; GBG 66): a randomised phase 2 trial. Lancet Oncol. 2014;15:747-56.

26. Loibl S, O'Shaughnessy J, Untch M, Sikov WM, Rugo HS, McKee MD, et al. Addition of the PARP inhibitor veliparib plus carboplatin or carboplatin alone to standard neoadjuvant chemotherapy in triple-negative breast cancer (BrighTNess): a randomised, phase 3 trial. Lancet Oncol. 2018;19:497-509.

27. Sharma P, Lopez-Tarruella S, García-Saenz JA, Khan QJ, Gomez HL, Prat A, et al. Pathological response and survival in triple-negative breast cancer following neoadjuvant carboplatin plus docetaxel. Clin Cancer Res. 2018;24:5820-9.

28. Sharma P, Kimler BF, O'Dea A, Nye L, Wang YY, Yoder R, et al. Randomized Phase II trial of anthracycline-free and anthracycline-containing neoadjuvant carboplatin chemotherapy regimens in stage I-III triple-negative breast cancer (NeoSTOP). Clin Cancer Res. 2021;27:975-82.

29. Yu KD, Ye FG, He M, Fan L, Ma D, Mo M, et al. Effect of adjuvant paclitaxel and carboplatin on survival in women with triple-negative breast cancer: a phase 3 randomized clinical trial. JAMA Oncol. 2020;6:1390-6.

30. Del Mastro L, De Placido S, Bruzzi P, De Laurentiis M, Boni C, Cavazzini G, et al. Fluorouracil and dose-dense chemotherapy in adjuvant treatment of patients with early-stage breast cancer: an open-label, $2 \times 2$ factorial, randomised phase 3 trial. Lancet. 2015;385:1863-72.

\section{ACKNOWLEDGEMENTS}

We thank all patients participating in the clinical trials and all study investigators of the PlanB and Success C study. Prior presentation: Presented in parts at the 53rd and 54th Annual Meeting of the American Society of Clinical Oncology, Chicago (2017 and 2018).

\section{AUTHOR CONTRIBUTIONS}

WJ and NH designed and conducted the study. AdG, TWPF, OG and NH drafted the article. AdG, UN, BR, AS, TF, HK, SK, ET, RW, AH, TR, PAF, OG and NH recruited patients, collected data and/or provided input for data interpretation. TWPF and RK were responsible for the statistical analysis. All authors approved the final article.

\section{FUNDING}

None was declared for the pooled analysis. The PlanB study was supported by Genomic Health, Amgen and Sanofi-Aventis, the Success $C$ study by Sanofi-Aventis, Chugai, Pfizer, GlaxoSmithKline and Janssen Diagnostics. Both studies were investigator-initiated and investigator-led and the bodies providing financial support played no part in the study design, data collection, data analysis, data interpretation, or writing of the report. The authors had full access to all of the data and had final 
responsibility for the decision to submit for publication. Open Access funding enabled and organized by Projekt DEAL.

\section{ETHICS APPROVAL AND CONSENT TO PARTICIPATE}

All participating patients provided written informed consent. Both trials were approved by the German ethics boards and conducted in accordance with the Declaration of Helsinki.

\section{CONSENT TO PUBLISH}

Not applicable.

\section{COMPETING INTERESTS}

AdG reports honoraria and/or travel costs from AstraZeneca, MSD, Roche, Tesaro, Novartis, Pfizer, Eisai and DaichiiSankyo. WJ reports grants and personal fees from Sanofi-Aventis, grants and personal fees from Novartis, grants and personal fees from Lilly, grants and personal fees from Pfizer, grants and personal fees from Roche, grants and personal fees from Chugai, grants and personal fees from AstraZeneca, grants and personal fees from MSD, grants and personal fees from Daiichi-Sankyo, from null, during the conduct of the study. TWPF reports honoraria from Novartis. UN reports grants from Amgen, Celgene, Genomic Health, and Hoffmann La Roche, nonfinancial support from Allgemeine Ortskrankenkasse (public medical insurance scheme); personal fees from Agendia, Amgen, AstraZeneca, Celgene, Genomic Health, Pfizer, ands Roche as well as accommodation expenses from Roche, Pfizer, and Celgene; and is a shareholder of WSG GmbH (Study Group). BR reports institutional funding from Sanofi-Aventis, Chugai, Pfizer, GlaxoSmithKline und Janssen Diagnostics. AS reports grants from Celgene, grants from Roche, grants from AbbVie, personal fees from Roche, personal fees from AstraZeneca, personal fees from Celgene, personal fees from Roche, personal fees from Roche, personal fees from Celgene, personal fees from Pfizer, personal fees from AstraZeneca, personal fees from Novartis, personal fees from MSD, personal fees from Tesaro, personal fees from Lilly, personal fees from Pfizer, other from Roche, outside the submitted work. TF reports Honoraria for lectures and/ or consulting from AstraZeneca, Daiichi-Sankyo, MSD, Novartis, Pfizer, Roche, TEVA, Onkowissen, HK reports honoraria from Roche Pharma, Astra Zenca, Exact Science, Pfizer, Lilly, Novartis. SK reports personal fees and/or travel expenses from Amgen, AstraZeneca, Celgene, Daiichi-Sankyo, F. Hoffmann-La Roche Ltd, Genentech, Genomic Health, Lilly, MSD Oncology, Novartis, Pfizer, PFM Medical, Somatex and Sonoscope. ET reports travel expenses by Sandoz. RW reports honoraria as advisor, consultant and/or speaker as well as travel grants from Agendia, Amgen, Aristo, AstraZeneca, Boeringer Ingelheim, Carl Zeiss, Celgene, Clinsol, Daiichi-Sankyo, Eisai, ExactSciences, Genomic Health, Glaxo Smith Kline, Hexal, Lilly, Medstrom Medical, MSD, Mundipharma, Nanostring, Novartis, Odonate, Paxman, Palleos, Pfizer, Pierre Fabre, PumaBiotechnolgogy, Riemser, Roche, Sandoz/Hexal, Seattle Genetics,
Tesaro Bio, Teva, Veracyte, Viatris. AH reports honoraria for lectures and/or consulting from Agendia, AstraZeneca, Daiichi-Sankyo, ExactSciences, Lilly, MSD, Novartis, Pierre Fabre, Pfizer, Roche, Sandoz/Hexal, Seattle Genetic, Teva. TR reports honoraria for lectures and/or advisory boards from AstraZeneca, Lilly, Novartis, Pfizer and Roche. PAF reports personal fees from Novartis, grants from Biontech, personal fees from Pfizer, personal fees from Daiichi-Sankyo, personal fees from AstraZeneca, personal fees from Eisai, personal fees from Merck Sharp \& Dohme, grants from Cepheid, personal fees from Lilly, personal fees from Pierre Fabre, personal fees from Seattle Genetics, personal fees from Roche, personal fees from Hexal, during the conduct of the study. OG reports honoraria for lectures and/or consulting from Roche, Novartis, Pfizer, Lilly, MSD, Amgen, Celgene/BMS, Exact Science, AstraZeneca, Pierre Fabre and travel expenses from Roche, Daiichi-Sankyo, Celgene. NH reports honoraria for lectures and/or consulting from AstraZeneca, Daiichi-Sankyo, Lilly, MSD, Novartis, Pierre Fabre, Pfizer, Roche, Sandoz/Hexal, Seattle Genetics. The remaining authors declare no competing interests.

\section{ADDITIONAL INFORMATION}

Supplementary information The online version contains supplementary material available at https://doi.org/10.1038/s41416-021-01690-6.

Correspondence and requests for materials should be addressed to Ameliede Gregorio.

Reprints and permission information is available at http://www.nature.com/ reprints

Publisher's note Springer Nature remains neutral with regard to jurisdictional claims in published maps and institutional affiliations.

Open Access This article is licensed under a Creative Commons Attribution 4.0 International License, which permits use, sharing, adaptation, distribution and reproduction in any medium or format, as long as you give appropriate credit to the original author(s) and the source, provide a link to the Creative Commons licence, and indicate if changes were made. The images or other third party material in this article are included in the article's Creative Commons licence, unless indicated otherwise in a credit line to the material. If material is not included in the article's Creative Commons licence and your intended use is not permitted by statutory regulation or exceeds the permitted use, you will need to obtain permission directly from the copyright holder. To view a copy of this licence, visit http://creativecommons. org/licenses/by/4.0/.

(c) The Author(s) 2022 